\title{
Case Report Antimicrobial susceptibility testing of Bordetella
pertussis in Taiwan prompted by a case of
pertussis in a paediatric patient
}

\author{
Shu-Man Yao, ${ }^{1}$ Gwo-Jen Liaw, ${ }^{2}$ Ying-Yan Chen, ${ }^{1}$ Meng-Hsiu Yen, ${ }^{3}$ \\ Ya-Hui Chen, ${ }^{1}$ Jung-Jung $\mathrm{Mu}^{1}$ and Chuen-Sheue Chiang ${ }^{1,4}$ \\ ${ }^{1}$ Research and Diagnostic Center, Centers for Disease Control, Taipei, Taiwan, ROC \\ ${ }^{2}$ Department of Life Sciences and Program in Genome Sciences, National Yang-Ming University, \\ Taipei, Taiwan, ROC \\ ${ }^{3}$ Departments of Pediatrics, Chang Gung Children's Hospital, Chang Gung Memorial Hospital and \\ Chang Gung University School of Medicine, Taoyuan, Taiwan, ROC \\ ${ }^{4}$ Center of General Education, National Taipei College of Nursing, Taipei, Taiwan, ROC
}

Correspondence

Chuen-Sheue Chiang

cschiang10@cdc.gov.tw

Received 24 April 2008

Accepted 11 August 2008

\begin{abstract}
In Taiwan, pertussis is a notifiable disease with a low incidence in recent years, and antimicrobial susceptibility testing for the causative agent, Bordetella pertussis, has not been reported to date. In May 2007, the Centers for Disease Control, Taiwan, was informed of a 1-month-old pertussis patient who did not respond to erythromycin treatment. In this study, we report the result of antimicrobial susceptibility testing performed for the suspected erythromycin-resistant isolate, as well as for an additional $27 \mathrm{~B}$. pertussis clinical isolates that represented almost all epidemiologically unrelated isolates obtained throughout Taiwan between 2003 and 2007. All isolates were fully susceptible to azithromycin, erythromycin, clarithromycin and trimethoprim/ sulfamethoxazole (MIC $\leqslant 0.047 \mu \mathrm{g} \mathrm{ml}^{-1}$ ). This result demonstrates the general susceptibility of $B$. pertussis to antimicrobial agents in vitro in Taiwan.
\end{abstract}

\section{Introduction}

Pertussis, a highly contagious respiratory disease caused by Bordetella pertussis, remains a significant cause of morbidity in children and more recently in adults (Broder et al., 2006; Celentano et al., 2005; Lin et al., 2007). For pertussis treatment and post-exposure prophylaxis, erythromycin has been the antimicrobial agent of choice (Bass, 1986; von König, 2005). Recently, azithromycin was shown to be as effective as and better tolerated than erythromycin for the treatment of pertussis in children (Langley et al., 2004). Due to lack of a standardized protocol for MIC determination, and the rarity of resistance to antimicrobial agents, antimicrobial susceptibility testing for $B$. pertussis clinical isolates is not routinely performed. However, there have been a few reports presenting the emergence of erythromycin-resistant $B$. pertussis isolates (Bartkus et al., 2003; Hill et al., 2000; Korgenski \& Daly, 1997; Lewis et al., 1995; Wilson et al., 2002). In Taiwan, pertussis is a notifiable disease with an incidence of less than 10 cases per million people in recent years (Lin et al., 2007). In May 2007, a 1-month-old paediatric pertussis patient was reported to the Centers for Disease Control, Taiwan, who did not respond to erythromycin treatment. In this study, we report the susceptibility testing result for this suspected erythromycin-resistant isolate, as well as for an additional
27 B. pertussis clinical isolates that represented almost all epidemiologically unrelated isolates obtained between 2003 and 2007 throughout Taiwan, to azithromycin, erythromycin, clarithromycin and trimethoprim/sulfamethoxazole.

\section{Case report}

The male infant was born on March 152007 with a gestational age of 34 weeks and a low birth weight of $2460 \mathrm{~g}$ due to premature rupture of the amniotic membrane; he was admitted to the observation room and given antibiotic treatment (ampicillin and cefotaxime) for 5 days, and then discharged. Blood and urine cultures were negative. On April 14 2007, the patient was admitted to the observation room again due to cyanosis, rhinorrhea, and poor activity and appetite for 1 day. Pneumonia was suspected with bilateral increased interstitial infiltrations shown on chest Xray, and an oxygen hood, and ampicillin and gentamicin were used to treat the patient. Two hours after admission, a sudden onset of apnea, bradycardia and cyanosis occurred; endotracheal intubation was performed, and the patient was transferred to the intensive care unit. Urine, blood and cerebrospinal fluid cultures were all negative. A haemogram, a cardiac echo and an electrocardiogram were normal. Brain 
echo showed bilateral mild periventricular flaring. Electroencephalography showed epileptiform discharge over the bilateral hemisphere; however, the patient did not have any convulsions. Luminal was prescribed because of suspected epilepsy. The patient was smoothly extubated on the fourth day of hospitalization. On April 27 2007, the patient was found to have a frequent cough, and subsequent apnoea, bradycardia and cyanosis. Pertussis was then suspected and erythromycin was added to the treatment regime. A nasopharyngeal swab from the patient was positive for B. pertussis by culture. So was a swab from his brother. The other close contacts were all negative, including his mother, father, one nurse who had fever and cough, and three other patients. All close contacts, including family members of the patient and all staff in the ICU, were given prophylactic antibiotics, either erythromycin or azithromycin. None of them developed pertussis-like symptoms. For the infant patient, the treatment with erythromycin was continued and the patient's symptoms improved gradually with a decreasing frequency of cyanosis. However, the patient's symptoms suddenly worsened with an increasing frequency of cyanosis on the twelfth day after using erythromycin. Therefore, treatment failure was suspected and antimicrobial susceptibility testing was requested.

\section{Methods}

Bacterial isolates and culture. Nasopharyngeal swabs in ReganLowe transport medium (Creative Microbiologicals) taken from the patient suspected of pertussis were delivered to the Centers for Disease Control, Taiwan, for culture confirmation. B. pertussis isolates were identified on Bordet-Gengou agar (Creative Microbiologicals), and characterized by biochemical reactions and slide agglutination test with Difco Bordetella antisera (Becton Dickinson). Isolates are stored in $15 \%$ glycerol or Protect beads (Technical Service Consultants) at $-80{ }^{\circ} \mathrm{C}$ until use.

Antimicrobial susceptibility testing. Antibiotic MICs were determined by Etest (AB Biodisk) as described by Galanakis et al. (2007) and Gordon et al. (2001). Bacteria were taken out from a $-80{ }^{\circ} \mathrm{C}$ freezer and subcultured onto Bordet-Gengou agar three times before susceptibility testing (Korgenski \& Daly, 1997). Mueller-Hinton agar plates supplemented with $5 \%$ horse blood were inoculated with bacterial suspension equal to a 0.5 McFarland turbidity standard prepared by direct bacterial colony suspension (CLSI, 2006). The antimicrobial agents tested were azithromycin, erythromycin, clarithromycin and trimethoprim/sulfamethoxazole. Plates were incubated at $35{ }^{\circ} \mathrm{C}$ in an ambient atmosphere for $72 \mathrm{~h}$. B. pertussis ATCC 9797 and Streptococcus pneumoniae ATCC 49619 were used as quality control strains both prior to and during testing of the 28 isolates. The former strain was expected to be sensitive to all four agents, and the latter strain was expected to fall in the ranges recommended by Clinical and Laboratory Standards Institute. The lowest concentration of the antimicrobial agent that completely inhibited bacterial growth is defined as the MIC. The $\mathrm{MIC}_{50}$ and $\mathrm{MIC}_{90}$ was the concentration of antimicrobial agent that inhibited growth of $50 \%$ and $90 \%$ bacterial isolates, respectively.

PFGE analysis. PFGE was performed for B. pertussis isolates with $\mathrm{XbaI}$ digestion as described by Yao et al. (2005). The resulting patterns were analysed using BioNumerics software, version 3.0 (Applied Maths). The Dice coefficient with an optimization setting of
$3 \%$ and a position tolerance of $1 \%$ was used to analyse the similarities among patterns. Cluster analysis was performed by UPGMA.

\section{Results and Discussion}

The suspected erythromycin-resistant isolate was obtained in April 2007 from a 1-month-old boy. An additional 27 B. pertussis clinical isolates representing almost all epidemiologically unrelated isolates in Taiwan from 2003 to 2007, including 7 isolates from 2003, 5 from 2004, 8 from 2005, 6 from 2006 and 1 from 2007 were also obtained. Together with the suspected erythromycin-resistant isolate, the 28 isolates in total were from 15 different cities and counties throughout the whole island of Taiwan, with 16, 6, 5 and 1 isolates in northern, central, southern and eastern Taiwan, respectively. A total of 16 of the 28 patients from whom the isolates came were female $(16 / 28,57.1 \%)$. The age distribution of the patients was: $16(57.1 \%)$ patients younger than 6 months, and $3(10.7 \%)$ patients each in the age groups of 7 months to 1 year, 2 to 10 years, 11 to 16 years and older than 16 years. Although vaccination information was not available, the patients younger than 6 months old apparently had not received a complete course of vaccination.

PFGE is performed routinely for all $B$. pertussis clinical isolates with $\mathrm{XbaI}$ digestion in our laboratory in order to monitor epidemiological trends by molecular methods. As shown in Fig. 1, among the 28 isolates tested for antimicrobial susceptibility, 18 isolates, including the suspected erythromycin-resistant one, belonged to PFGE group IIIb, which has been the most prevalent PFGE group since 2001 in Taiwan (Yao et al., 2005). Nine isolates belonged to PFGE group IIIa, which was the most prevalent PFGE group before being replaced by group IIIb in 2001. One isolate collected in 2003 belonged to PFGE group II, which group was last seen in 2003.

The MICs of azithromycin, erythromycin, clarithromycin and trimethoprim/sulfamethoxazole were determined (Table 1). All the clinical B. pertussis isolates, including the suspected erythromycin-resistant isolate, appeared to be susceptible to all four antimicrobial agents. All MICs were $\leqslant 0.047 \mu \mathrm{g} \mathrm{ml}^{-1}$. The MIC 50 was $0.016 \mu \mathrm{g} \mathrm{ml}^{-1}$ for all four antimicrobial agents. The MIC 90 was $0.016 \mu \mathrm{g} \mathrm{ml}^{-1}$ for azithromycin, and $0.023 \mu \mathrm{g} \mathrm{m}{ }^{-1}$ for erythromycin, clarithromycin and trimethoprim/sulfamethoxazole. For erythromycin, a MIC $>0.016 \mu \mathrm{g} \mathrm{ml}^{-1}$ was observed only in three isolates. These three isolates also had a MIC $>0.016$ $\mu \mathrm{g} \mathrm{ml}^{-1}$ for clarithromycin (all three isolates), azithromycin (one isolate) and trimethoprim/sulfamethoxazole (one isolate). For the suspected erythromycin-resistant isolate, the MICs for azithromycin, erythromycin and clarithromycin were $0.016 \mu \mathrm{g} \mathrm{ml}^{-1}$, and the MIC for trimethoprim/ sulfamethoxazole was $0.023 \mu \mathrm{g} \mathrm{ml}^{-1}$. Our MIC values were comparable with those published by Galanakis et al. (2007), in the range of $0.016-0.19,0.016-0.094$ and $0.002-0.064 \mu \mathrm{g} \mathrm{ml}^{-1}$ for azithromycin, erythromycin and 
Dice (Opt:3.00\%) (Tol 1.0\%-1.0\%) (H>0.0\% S>0.0\%) [0.0\%-100.0\%]

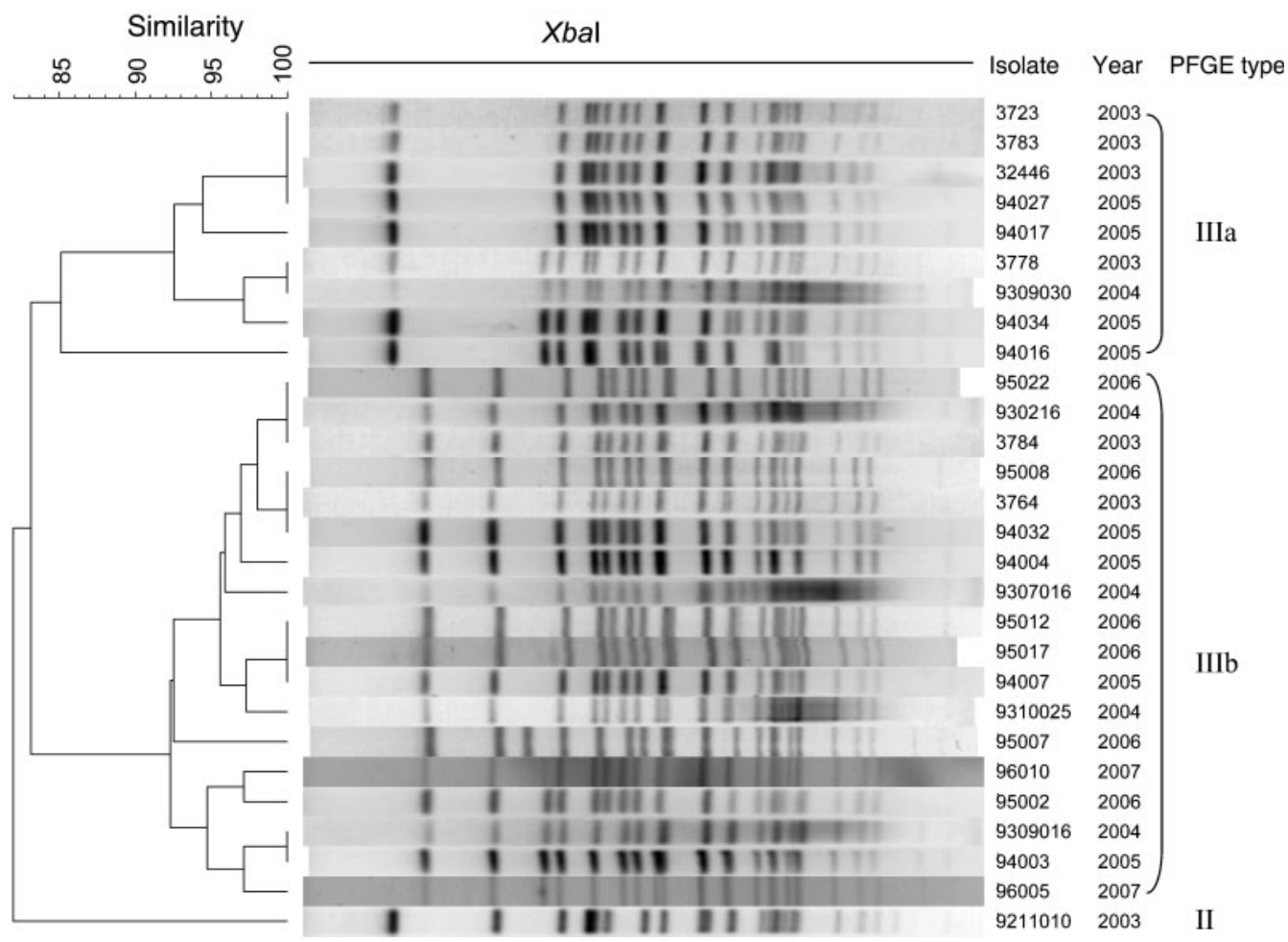

Fig. 1. PFGE patterns of $28 \mathrm{~B}$. pertussis clinical isolates with $\mathrm{Xbal}$ digestion.

trimethoprim/sulfamethoxazole, respectively. For the quality control strains, MICs of azithromcin, erythromycin, clarithromycin and trimethoprim/sulfamethoxazole were as expected: $0.016,0.016,0.016$ and $0.002 \mu \mathrm{g} \mathrm{ml} \mathrm{ml}^{-1}$, respectively, for $B$. pertussis ATCC 9797; and 0.125, 0.064, 0.032 and $0.125 \mu \mathrm{g} \mathrm{ml}^{-1}$, respectively, for S. pneumoniae ATCC 49619.

The paediatric pertussis patient who prompted this investigation eventually recovered and his slow response to medication was rationalized as the cause for the initial therapeutic failure. Similarly, in 2006, a 4-month-old boy born at 26 weeks of pregnancy was documented as exhibiting treatment failure for pertussis in France (Bonacorsi et al., 2006). In the latter case, since the $B$. pertussis isolate was susceptible to erythromycin, the authors' explanation for the treatment failure was the infant's immunological immaturity. They further suggested that the gold standard of treatment with erythromycin for pertussis is likely to be insufficient in premature infants.

Our results demonstrated the general susceptibility of $B$. pertussis to antimicrobial agents in vitro in Taiwan. Nevertheless, even though they represented almost all epidemiologically unrelated isolates that were collected throughout Taiwan from 2003 to 2007, the number of isolates in our study was small. Since the first case of erythromycin-resistant $B$. pertussis was identified in Arizona, USA, in 1994 (Lewis et al., 1995), only four more cases were reported in Minnesota, California, Arizona and Utah, USA, (Bartkus et al., 2003; Hill et al., 2000; Korgenski \& Daly, 1997). After screening 1030 B pertussis isolates, 5

Table 1. Antimicrobial susceptibility of $28 \mathrm{~B}$. pertussis isolates by the Etest method

\begin{tabular}{|lccc|}
\hline Antimicrobial agent & $\mathbf{M I C}$ range $\left(\boldsymbol{\mu g} \mathbf{~ m l}^{-\mathbf{1}}\right)$ & $\mathbf{M I C}_{\mathbf{5 0}}\left(\boldsymbol{\mu} \mathbf{~} \mathbf{~ m l}^{-\mathbf{1}}\right)$ & $\mathbf{M I C}_{\mathbf{9 0}}\left(\boldsymbol{\mu g} \mathbf{~ m l}^{\mathbf{- 1}}\right)$ \\
\hline Azithromycin & $0.016-0.023$ & 0.016 & 0.016 \\
Erythromycin & $0.016-0.023$ & 0.016 & 0.023 \\
Clarithromycin & $0.016-0.047$ & 0.016 & 0.023 \\
Trimethoprim/ & $0.012-0.032$ & 0.016 & 0.023 \\
sulfamethoxazole & & & \\
\hline
\end{tabular}


more isolates with a heterogeneous erythromycin-resistance phenotype were found, leading to the conclusion that the occurrence rate of erythromycin-resistant $B$. pertussis is less than $1 \%$ (Wilson et al., 2002). Such a low rate probably could explain why no resistant isolates were found in our study and argue for maintaining the screening for resistance among $B$. pertussis clinical isolates that may yet emerge.

\section{Acknowledgements}

This work was supported in part by grants DOH96-DC-2022 from the Centers for Disease Control, Department of Health, Taiwan, and 950324-19-F01 from the National Science Council, Executive Yuan, Taiwan.

\section{References}

Bartkus, J. M., Juni, B. A., Ehresmann, K., Miller, C. A., Sanden, G. N., Cassiday, P. K., Saubolle, M., Lee, B., Long, J. \& other authors (2003). Identification of a mutation associated with erythromycin resistance in Bordetella pertussis: implications for surveillance of antimicrobial resistance. J Clin Microbiol 41, 1167-1172.

Bass, J. W. (1986). Erythromycin for treatment and prevention of pertussis. Pediatr Infect Dis 5, 154-157.

Bonacorsi, S., Farnoux, C., Bidet, P., Caro, V., Aizenfisz, S., Benhayoun, M., Aujard, Y., Guiso, N. \& Bingen, E. (2006). Treatment failure of nosocomial pertussis infection in a very-lowbirth-weight neonate. J Clin Microbiol 44, 3830-3832.

Broder, K. R., Cortese, M. M., Iskander, J. K., Kretsinger, K., Slade, B. A., Brown, K. H., Mijalski, C. M., Tiwari, T., Weston, E. J. \& other authors (2006). Preventing tetanus, diphtheria, and pertussis among adolescents: use of tetanus toxoid, reduced diphtheria toxoid and acellular pertussis vaccines. Recommendations of the Advisory Committee on Immunization Practices (ACIP). MMWR Recomm Rep 55, 1-34.

Celentano, L. P., Massari, M., Paramatti, D., Salmaso, S. \& Tozzi, A. E. on behalf of the EUVAC-NET group (2005). Resurgence of pertussis in Europe. Pediatr Infect Dis J 24, 761-765.
CLSI (2006). Performance Standards for Antimicrobial Disk Susceptibility Test, 9th edn, approved standard M2-A9. Wayne, PA: Clinical and Laboratory Standards Institute.

Galanakis, E., Englund, J. A., Abe, P. \& Qin, X. (2007). Antimicrobial susceptibility of Bordetella pertussis isolates in the state of Washington. Int J Antimicrob Agents 29, 609-611.

Gordon, K. A., Fusco, J., Biedenbach, D. J., Pfaller, M. A. \& Jones, R. N. (2001). Antimicrobial susceptibility testing of clinical isolates of Bordetella pertussis from northern California: report from the SENTRY Antimicrobial Surveillance Program. Antimicrob Agents Chemother 45, 3599-3600.

Hill, B. C., Baker, C. N. \& Tenover, F. C. (2000). A simplified method for testing Bordetella pertussis for resistance to erythromycin and other antimicrobial agents. J Clin Microbiol 38, 1151-1155.

Korgenski, E. K. \& Daly, J. A. (1997). Surveillance and detection of erythromycin resistance in Bordetella pertussis isolates recovered from a pediatric population in the Intermountain West region of the United States. J Clin Microbiol 35, 2989-2991.

Langley, J. M., Halperin, S. A., Boucher, F. D., Smith, B. \& the Pediatric Investigators Collaborative Network on Infections in Canada (PICNIC) (2004). Azithryomycin is as effective as and better tolerated than erythromycin estolate for the treatment of pertussis. Pediatrics 114, e96-e101.

Lewis, K., Saubolle, M. A., Tenover, F. C., Rudinsky, M. F., Barbour, S. D. \& Cherry, J. D. (1995). Pertussis caused by an erythromycinresistant strain of Bordetella pertussis. Pediatr Infect Dis J 14, 388-391.

Lin, Y. C., Yao, S. M., Yan, J. J., Chen, Y. Y., Chiang, C. S., Wu, H. S. \& Li, S. Y. (2007). Epidemiological shift in the prevalence of pertussis in Taiwan: implications for pertussis vaccination. J Med Microbiol 56, 533-537.

von König, C.-H. W. (2005). Use of antibiotics in the prevention and treatment of pertussis. Pediatr Infect Dis J 24, S66-S68.

Wilson, K. E., Cassiday, P. K., Popovic, T. \& Sanden, G. N. (2002). Bordetella pertussis isolates with a heterogeneous phenotype for erythromycin resistance. J Clin Microbiol 40, 2942-2944.

Yao, S. M., Lin, Y. C., Chou, C. Y., Chen, Y. Y., Hsiao, M. J., Chen, H. Y., Yan, J. J., Su, H. P. \& Li, S. Y. (2005). Antigenic divergence of Bordetella pertussis isolates in Taiwan. J Clin Microbiol 43, 5457-5461. 